\title{
An experimental vital signs detection radar using low-IF heterodyne architecture and single-sideband transmission
}

Jensen, Brian Sveistrup; Johansen, Tom Keinicke; Yan, Lei

Published in:

Proceedings of 2013 IEEE International Wireless Symposium (IWS)

Link to article, DOI:

10.1109/IEEE-IWS.2013.6616752

Publication date:

2013

Link back to DTU Orbit

Citation (APA):

Jensen, B. S., Johansen, T. K., \& Yan, L. (2013). An experimental vital signs detection radar using low-IF heterodyne architecture and single-sideband transmission. In Proceedings of 2013 IEEE International Wireless Symposium (IWS) IEEE. https://doi.org/10.1109/IEEE-IWS.2013.6616752

\section{General rights}

Copyright and moral rights for the publications made accessible in the public portal are retained by the authors and/or other copyright owners and it is a condition of accessing publications that users recognise and abide by the legal requirements associated with these rights.

- Users may download and print one copy of any publication from the public portal for the purpose of private study or research.

- You may not further distribute the material or use it for any profit-making activity or commercial gain

- You may freely distribute the URL identifying the publication in the public portal 


\title{
An Experimental Vital Signs Detection Radar using Low-IF Heterodyne Architecture and Single-Sideband Transmission
}

\author{
Brian Sveistrup Jensen ${ }^{1,2}$, Tom K. Johansen ${ }^{1}$, Lei Yan ${ }^{1}$ \\ ${ }^{1}$ Technical University of Denmark, Kgs. Lyngby, DK-2800, Denmark \\ ${ }^{2}$ Thrane \& Thrane, Kgs. Lyngby, DK-2800, Denmark \\ Email: tkj@elektro.dtu.dk
}

\begin{abstract}
In this paper an experimental X-band radar system, called DTU-VISDAM, developed for the detection and monitoring of human vital signs is described. The DTUVISDAM radar exploits a low intermediate frequency (IF) heterodyne RF front-end architecture and single-sideband (SSB) transmission for easier and more reliable extraction of the vital signs. The hardware implementation of the proposed low-IF RF front-end architecture and associated IF circuitry is discussed. Furthermore, the signal processing and calibration steps necessary to extract the vital signs information measured on a human subject are described.

Index Terms - Human vital signs, low-IF architecture, radar, single-sideband transmission.
\end{abstract}

\section{INTRODUCTION}

The measurement of human vital signs by wireless technology has been of great interest since the first sensors were demonstrated in the early 1970's [1]. Essentially, these sensors are based upon microwave radar detection where the mechanically movement of the chest of the human subject results in a measurable change in the returned radar signal. By subsequent data processing it is possible to identify the heartbeat and respiration rates.

Despite an extensive research effort in non-contact vital signs detection (VSD) radar within the past decade [2-3], these wireless sensors still lack widespread acceptance in the health care community. A major problem is that the faint return signal due to the physiological chest movement is difficult to separate from the much stronger interference signals caused by random body movements and unwanted reflections from static objects within the antenna beam. Furthermore, the received low-frequency baseband signal ranging from 0.1 to $3.3 \mathrm{~Hz}$ may be buried in $1 / f-$ noise coming from the electronic components, in particular the amplifiers and mixers in the receiver chain.

To overcome the limitation experienced with present VSD radars, which are mainly based on the direct conversion (homodyne) architecture, the low-IF heterodyne architecture was recently proposed [4]. Although the hardware is slightly more complex, the lowIF heterodyne architecture reduces or completely eliminates the problems with $1 /$ f-noise, DC offset errors, and I/Q channel mismatches. In [5], the authors showed

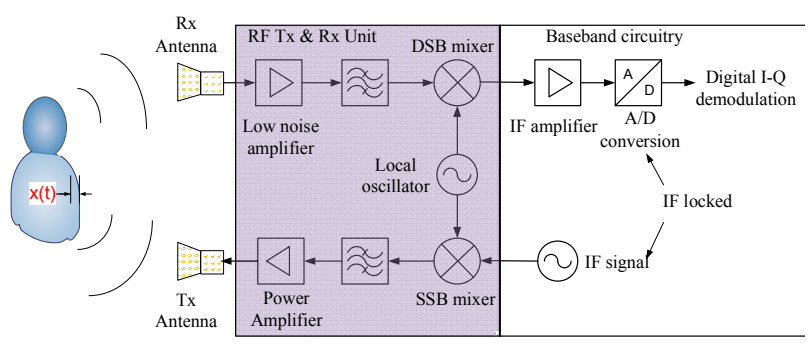

Fig. 1. The low-IF heterodyne VSD radar architecture. The human subject under test is also shown.

theoretically that the low-IF heterodyne architecture suffers from null-point detection distances similar to that found in single chancel direct conversion VSD radars [3]. To avoid these null-point detection distances the use of single-sideband transmission was proposed and an instrument based VSD radar was built to demonstrate the concept.

Recently, a complete experimental X-band radar system for vital signs detection and monitoring, called DTUVISDAM, has been implemented at the Technical University of Denmark (DTU). The aim of this radar is to demonstrate the benefits of the proposed low-IF heterodyne architecture with single-sideband transmission for VSD radars. To the best of the author's knowledge the DTU-VISDAM radar presents the first non-instrument based VSD radar based upon the low-IF heterodyne architecture with single-sideband transmission.

\section{LOW-IF HETERODYNE ARCHITECTURE}

A simplified block diagram for a VSD radar using a low-IF heterodyne architecture is shown in fig. 1. The shown architecture use an up-converter sidestep configuration with a mixer and an IF generator. A downconversion sidestep configuration is also possible. Going through the signal theory for these two configurations reveals that they are essentially identical in operation. Common to both configurations is the locking (coherency) between the sampling circuit and the IF generation circuit. This is important in order to suppress the phase noise generated in the IF generator circuit. 


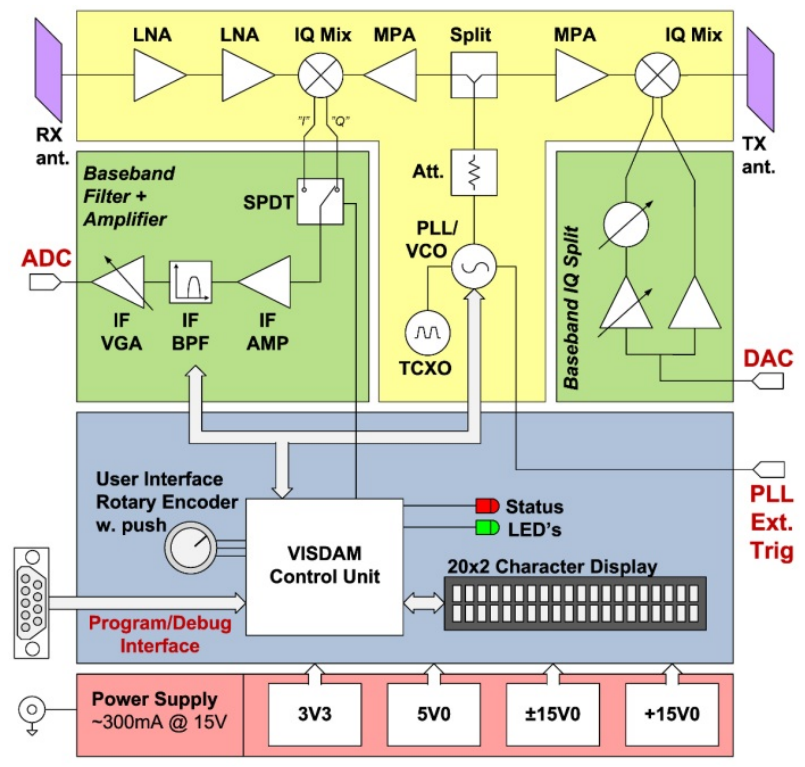

Fig. 2. Block diagram of the DTU-VISDAM radar.

One of the drawbacks of the architecture in Fig. 1 is an increase of spectral components in the sidestep signal, owing to additional mixing products. To this end, one of the most important design aspects is that an SSB signal is needed for transmission (or down-conversion). This requires that the sidestep mixer is configured as a slightly more complicated circuit, i.e. as an image-reject mixer (IRM). In [5], the authors presented a detailed analysis highlighting the difference between using an SSB sidestep mixer rather than the simpler double-sideband (DSB) sidestep mixer. To emphasize the importance of this choice, the IF signals obtained by using either techniques are given here in compact form as

$$
S_{D S B}(t)=\cos \left(\frac{4 \pi}{\lambda_{R F}} x(t)+\frac{4 \pi}{\lambda_{R F}} d_{0}\right) \cdot \cos \left(\omega_{I F} t\right)
$$

for the DSB case and

$$
S_{S S B}(t)=\cos \left(\omega_{I F} t+\frac{4 \pi}{\lambda_{R F}} x(t)+\frac{4 \pi}{\lambda_{R F}} d_{0}\right)
$$

for the SSB case where ${ }_{\mathrm{IF}}=2 \mathrm{f}_{\mathrm{IF}}$ is the IF angular frequency, $x(t)$ represents the chest wall movement due to heart- and respiration activity, $\mathrm{d}_{0}$ is the nominal distance to the target, and ${ }_{\mathrm{RF}}$ is the resultant wavelength associated with the up-converted RF signal. Similar wavelength ${ }_{\mathrm{RF}}$ is assumed for both the upper and lower sidebands due to the low-IF frequency. It can be seen from (1) that the IF signal obtained using a DSB sidestep mixer is a sine wave at the IF frequency with its envelope modulated by the chest wall movement. Therefore null-points and optimumpoints will be present and determined by the nominal distance, $d_{o}$, to the subject under test. In the case of an

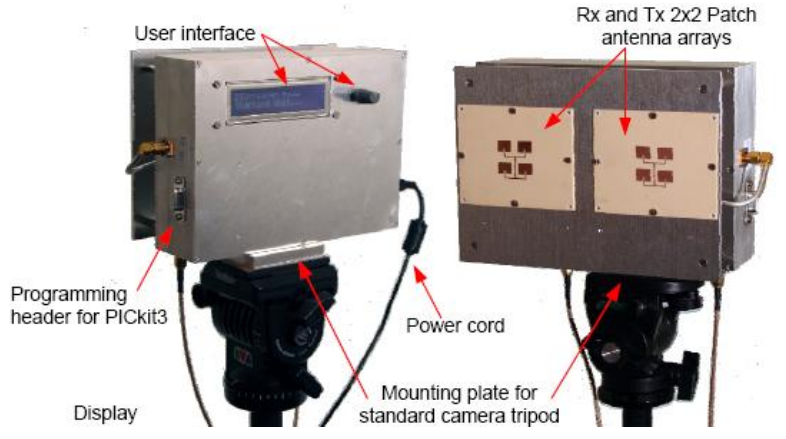

Fig. 3. Front and back view of the DTU-VISDAM radar assembly.

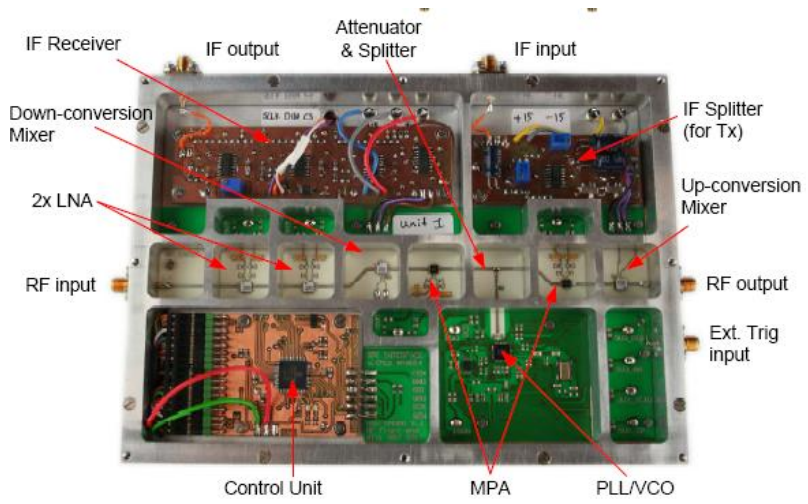

Fig. 4. Photograph of DTU-VISDAM's front-end electronics.

SSB sidestep mixer, (2) shows that the modulation is placed onto the phase of the IF signal meaning that the envelope is no longer modulated by the variation in distance, i.e. null-detection points have been eliminated.

\section{THE DTU-VISDAM RADAR SYSTEM}

A block diagram of the DTU-VISDAM radar is shown in Fig. 2. The top part consists of an RF transceiver and two IF parts for the transmitter and receiver, respectively, while the bottom part consists of the control unit and the power supply unit. The IF parts handles the IF signal amplification and filtering in the receive path as well as IF signal split in the transmission path. The IF signal split generate signals with 90 degrees phase difference to be fed into a Hittite I/Q mixer (model: HMC5271LC4) which effectively operates as an image reject mixer (IRM). This generates the single-sideband signal needed for transmission. The amplitude and phase of the split IF can be adjusted independently for best image suppression of the transmitted signal. The antennas are implemented as $2 \times 2$ microstrip patch antenna arrays with a measured gain of approximately $11.1 \mathrm{dBi}$ and 58 degrees beamwidth. A key component in the DTU-VISDAM radar is the Hittite PLL with integrated VCO (model: HMC769LP6CE) which serves as the common $\mathrm{RF}$ oscillator with a frequency set in the range from 9.05 to $10.15 \mathrm{GHz}$. The mixer LO inputs are provided through a set of medium 
power amplifiers from Hittite (model: HMC451LP3). A combination of a resistive attenuator and a resistive splitter provides an option for tailoring the LO input power to the mixers. With this setup it is possible to provide up to $16 \mathrm{dBm}$ of $\mathrm{LO}$ power to the mixers. The receive path of the RF front-end consists of two cascaded low-noise amplifiers (LNA's) from Hittite (model: HMC564LC4) followed by yet another Hittite I/Q mixer (model: HMC5271LC4). Only a single IF output (I or Q channel) is selected from the receive mixer for further processing. This is determined by a Vishay SPDT switch (model: DG403). The IF frequency is chosen to be $1 \mathrm{kHz}$ as this is easy to both generate and sample digitally. Coherent operation is ensured by using the same clock source for both IF signal generation and sampling.

Fig 3 shows the front and back views of the assembled DTU-VISDAM radar. The front view provides a custom tailored user interface for selection of frequency, IF channel selection (I or $\mathrm{Q}$ out of the receive mixer), and signal amplification. A photograph of the front-end electronics is shown in Fig 4. The RF design comprises a single PCB containing all sub circuits. To increase the isolation between them the entire RF part is subdivided into 20x20x15 mm (width, length, height) compartments such that the cavity resonance frequency is located well above the radar operation frequency. The performance of the complete radar unit is summarized in table I.

TABLE I.

Performance parameters of the VISDAM radar unit

\begin{tabular}{|l|c|c|}
\hline & Performance & $\mathrm{Units}$ \\
\hline $\begin{array}{l}\text { RF Bandwidth (RF radar front- } \\
\text { end) }\end{array}$ & $9.05-10.15$ & $\mathrm{GHz}$ \\
\hline $\begin{array}{l}\text { RF Bandwidth (inclusive } \\
\text { antennas) }\end{array}$ & $9.235-9.455$ & $\mathrm{GHz}$ \\
\hline Image Reject Ratio (output) & $>45$ & $\mathrm{~dB}$ \\
\hline Transmit Power & -40 to 0 & $\mathrm{dBm}$ \\
\hline Antenna Gain (both Tx and Rx) & 11.1 & $\mathrm{dBi}$ \\
\hline Antenna Cross Talk (Tx-to-Rx) & $<-65$ & $\mathrm{~dB}$ \\
\hline IF Center Frequency & 1.0 & $\mathrm{kHz}$ \\
\hline IF Bandwidth & 3.0 & $\mathrm{kHz}$ \\
\hline Tx-Rx Isolation (RF front-end) & 75 & $\mathrm{~dB}$ \\
\hline Supply Voltage & 15.0 & $\mathrm{~V}$ \\
\hline Power Consumption & 5.25 & $\mathrm{~W}$ \\
\hline
\end{tabular}

\section{EXPERIMENTAL RESULTS}

\section{A. Digital I/Q Demodulation and Calibration}

The output signal of the DTU-VISDAM radar is a timedomain sinusoidal signal at $1 \mathrm{kHz}$ with a nearly constant envelope due to the single-sideband transmission and with a phase modulated by the heartbeat and respiration activities from the subject under test. The extraction of the vital signs information from the sampled low-IF signal is

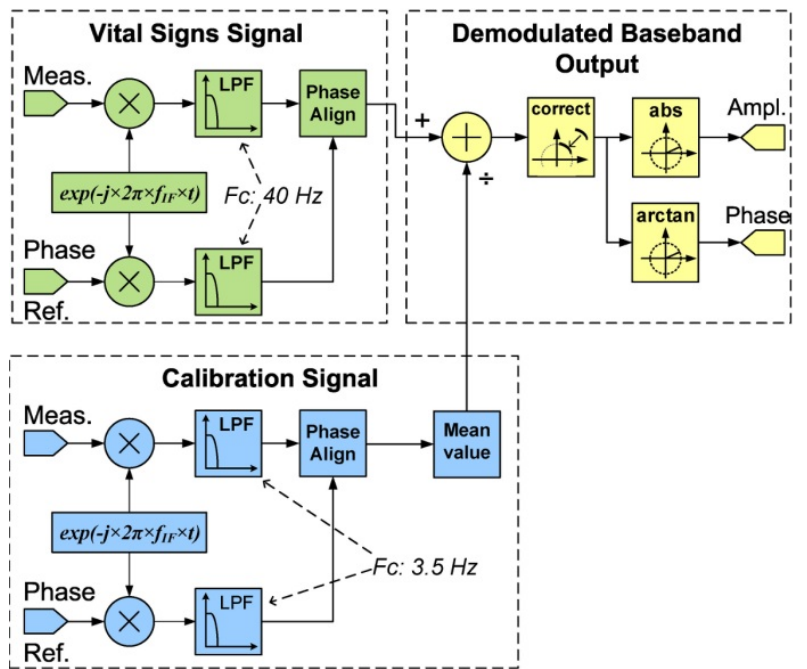

Fig. 5. General procedure for amplitude and phase extraction through digital I/Q demodulation and calibration.

based on a digital I/Q demodulation technique. The general procedure for extraction of the amplitude and phase information is outlined in Fig. 5. In general, two measurements are used, i.e. a vital signs measurement and a calibration measurement, both of which include a reference signal for phase alignment between measurements taken at different times. The phase reference is simply a loop-back of the outputted IF signal. The calibration is performed using a microwave absorber in front of the radar to measure the cross-talk.

The first step in the demodulation process is to downconvert the input signal to an I/Q complex baseband signal. Mathematically this first step can be written as,

$$
\mathrm{I}(\mathrm{n})+\mathrm{j} \cdot \mathrm{Q}(\mathrm{n})=\mathrm{S}(\mathrm{n}) \cdot \mathrm{e}^{-\mathrm{j} 2 \pi \mathrm{t}(\mathrm{n}) \mathrm{f}_{\mathrm{IF}}}
$$

where $S(n)$ represents the sampled IF data and $t(n)$ is the time vector. Once the I/Q baseband signal is obtained, a low pass filter (LPF) of the FIR type is applied to the data. After low pass filtering, the complex signals from both the calibration and VSD signal, are phase corrected using the reference phase acquired during each measurements. This step ensures that the calibration data can be properly referenced to the VSD signal. As the calibration data are not modulated they resemble ideally a single point DC offset in the I/Q plane. The mean value of the calibrated data is calculated to remove noise and subtracted from the VSD data.

The calibrated VSD data is still not sufficient for proper detection as the influence from clutter signals is too high. Therefore, the calibrated signal is run through a "best circle fitting" algorithm. It fits the data to a circle with origin near $(0,0)$ by running through a number of DC offset points around the calibrated data and finding the point at which the standard deviation of the amplitude signal is lowest. Figure 6 shows an I/Q plot after 


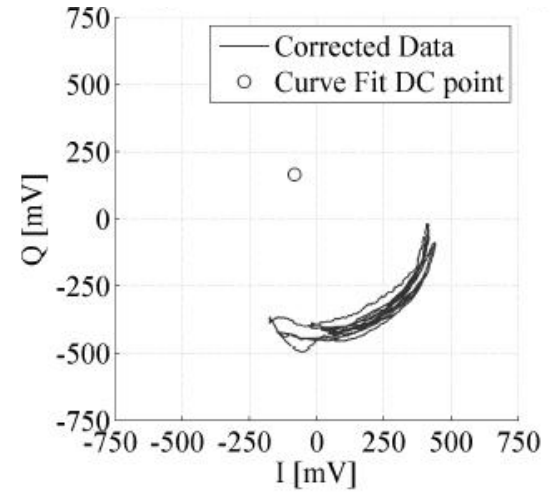

Fig. 6. I/Q plot after calibration and curve-fitting.

calibration and curve fitting. The signal amplitude and phase can now be extracted based arctangent demodulation performed on the corrected I/Q data [6].

\section{B. Analysis of Extracted Vital Signs Signals}

The analysis of the extracted vital signs signals begins with filtering to separate the signals containing respiration or heartbeat signals. The heartbeat signal is selected as the output from a high pass filter with a cut-off frequency around $0.7 \mathrm{~Hz}$ while the respiration signal is taken as the output signal from a low-pass filter with cut-off frequency of $0.6 \mathrm{~Hz}$. Having isolated the heartbeat signal, a moving window is applied for tracking of the heart rate. For the extraction of the heart rate, two different methods could be applied. The first method is based on the search of the highest spectral peak in the FFT spectrum. The second method calculates the auto-correlation of the time signal and finds the periodicity in the resulting signal. As the signals are not truly sinusoidal in nature, the latter analysis technique is applied. The analysis steps for evaluation and tracking of the respiration rate is identical to those described above.

Fig. 7 shows the extracted respiration and heartbeat signals along with the heartbeat reference signal obtained with a finger pressure transducer. The extracted respiration rate is approximately 10 breaths/minute and the heart rate is around 60 beats/minute. The detected heart rate is tracked within $10 \%$ of the reference at all times.

\section{CONCLUSION}

In this paper an experimental X-band radar for vital signs detection and monitoring was described. The VSD radar uses a low-IF architecture with single-sideband transmission. The operation of the VSD radar together with the implemented signal processing methods was verified experimentally on a human subject.
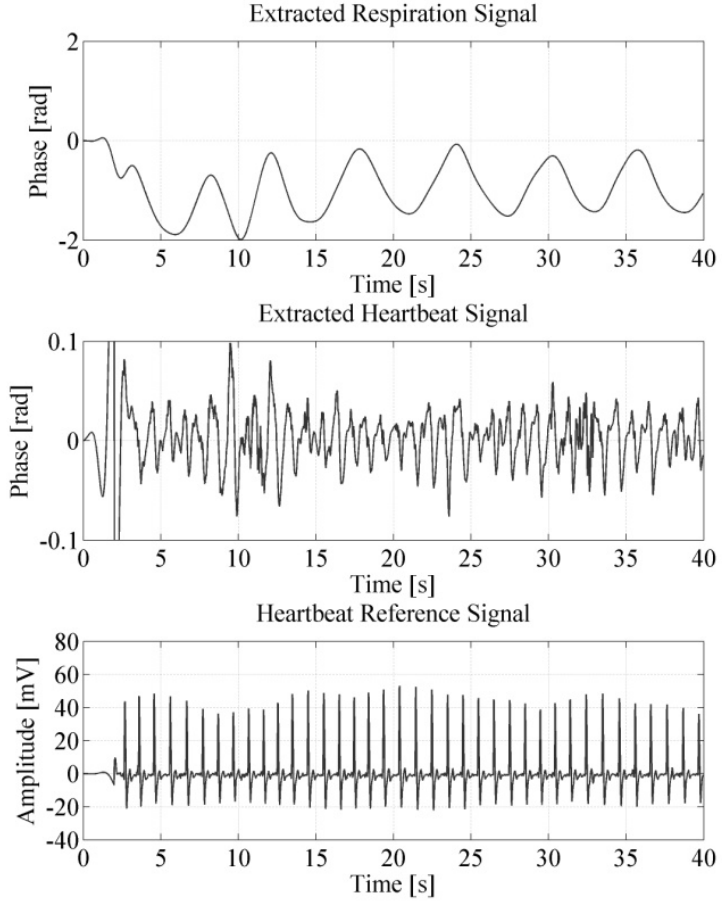

Fig. 7. Extracted respiration and heartbeat signals. Bottom plot shows the heartbeat reference signal obtained with a finger pressure transducer.

\section{ACKNOWLEDGEMENT}

The authors wish to acknowledge the Danish fond "Radiopartsfonden" for financial support.

\section{REFERENCES}

[1] J. C. Lin, "Microwave Movement Sensing of Physiological and Volume Change: A Review," Bioelectromagnetics, vol. 13 , issue 6, pp. 557-565, 1992.

[2] A. D. Droitcour et al., "Range Correlation and I/Q Performance Benefits in Single-Chip Silicon Doppler Radars for Noncontact Cardiopulmonary Monitoring," IEEE Trans. Microwave Theory and Tech., vol. 52, no. 3, pp. 838-848, March 2004 .

[3] Y. Xiao et al., "Frequency-Tuning Technique for Remote Detection of Heartbeat and Respiration Using Low-Power Double-Sideband Transmission in the Ka-band," IEEE Trans. Microwave Theory and Tech., vol. 54, no. 5, pp. 2023-2032, May 2006.

[4] C. Gu et. al, "Instrument-Based Noncontact Doppler Radar Vital Sign Detection System Using Heterodyne Digital Quadrature Demodulation Architecture," IEEE Trans. Inst. And Meas. ,vol. 59, no. 6, pp. 1580-1587, June 2010.

[5] B. S. Jensen et. al, "Vital Signs Detection Radar using Low Intermediate-Frequency Architecture and Single-Sideband Transmission," European Microwave Conf., Nov. 2012.

[6] B.-K. Park, O. B.-Lubecke, and V. Lubecke, "Arctangent Demodulation With DC Offset Compensation in Quadrature Doppler Radar Receiver Systems," IEEE Trans Microwave Theory and Tech.,vol 55, no. 5, May 2007. 